\title{
Aneurysmal bone cyst of the maxilla rare presentation with radiological and pathological correlation
}

\author{
Bharat Bhushan Sharma1, Priya Ramchandran², Sandeep Sharma ${ }^{3}$, Shweta Sharma ${ }^{4}$ \\ ${ }^{1}$ Department of Radio Diagnosis, PGIMER and Dr. RML Hospital, New Delhi 110001, India. \\ ${ }^{2}$ Intensive Care Unit and Anesthesiology, Heartlands Hospital, B912AF, Brimingham, UK. \\ ${ }^{3}$ Pain Management and Anesthesiology SGT Medical College, Gurgaon 122505, Haryana, India. \\ ${ }^{4}$ ENT and Head and Neck Surgery, PGIMER and Dr. RML Hospital, New Delhi 110001, India.
}

Address for correspondence: Dr. Bharat Bhushan Sharma, Department of Radio Diagnosis, PGIMER and RML Hospital, B-32, Nivedita Kunj Sector 10 R.K. Puram, New Delhi 110001, India. E-mail: bbhushan986@gmail.com

\begin{abstract}
The incidence of aneurysmal bone cyst in the maxillofacial region is rare and may remain undiagnosed for a long period prior to becoming symptomatic. This may cause associated issues secondary to compression by extending to the surrounding vital anatomical areas. An aggressive course can lead to bony destruction with intracranial extension. We present a case of a 23-year-old man who presented with bilateral exorbitism with nasal obstruction.
\end{abstract}

Key words:

Aggressive course, aneurysmal bone cyst, bone destruction, intracranial extension

\section{INTRODUCTION}

Aneurysmal bone cysts $(\mathrm{ABC})$ are typically found in long bones and the spine, but rarely can be seen in the craniofacial region. The incidence is $2 \%$ and $1.3 \%$ in the craniofacial region and maxillary region, respectively. ${ }^{[1,2]}$ These benign, expansile bony tumors of unknown etiology are often referred to as aneurysmal bone tumors rather than cysts because of their aggressive behavior.

\section{CASE REPORT}

A 23-year-old man presented with a history of anosmia, the sensation of a blocked nose with mild pain that had been increasing over 6 months, and a gradual increase in bilateral exorbitism. The patient denies any history of fever, trauma, epistaxis, or oral bleeding. His

\begin{tabular}{|l|l|}
\multicolumn{2}{|c|}{ Access this article online } \\
\hline Quick Response Code: & Website: \\
\hline & www.parjournal.net \\
\cline { 2 - 2 } & \\
\hline
\end{tabular}

physical examination was remarkable for a brownish bulge in the nasopharynx. The patient underwent computerized tomography (CT) and magnetic resonance imaging (MRI) examinations. Noncontrast computerized tomography (NCCT) of the nasopharynx region showed an expansile cystic lesion in the maxillary region bulging into the nasopharynx [Figure 1]. There was no cortical break [Figures 2 and 3]. Multiple fluid levels were seen within the lesion [Figures 4 and 5]. Cytology following fine-needle aspiration was performed, and a cytological diagnosis of $A B C$ of the maxilla was given. The patient underwent enucleation of the mass. Histopathological examination further confirmed the diagnosis of a maxillary $\mathrm{ABC}$, with cystic spaces filled with blood, and without an endothelial lining along with osteoclast giant cells [Figure $6 a$ and b]. There were no surgical complications, and the patient was advised to follow-up in 3 months. His recovery has been uneventful.

\section{DISCUSSION}

The maxilla is a rare site of $\mathrm{ABC}$. It is neither an aneurysm nor a cyst. The World Health Organization (WHO) defines an $\mathrm{ABC}$ as an "expanding osteolytic lesion consisting of blood-filled spaces of variable size separated by connective tissue septa containing trabeculae of osteoid tissue and 


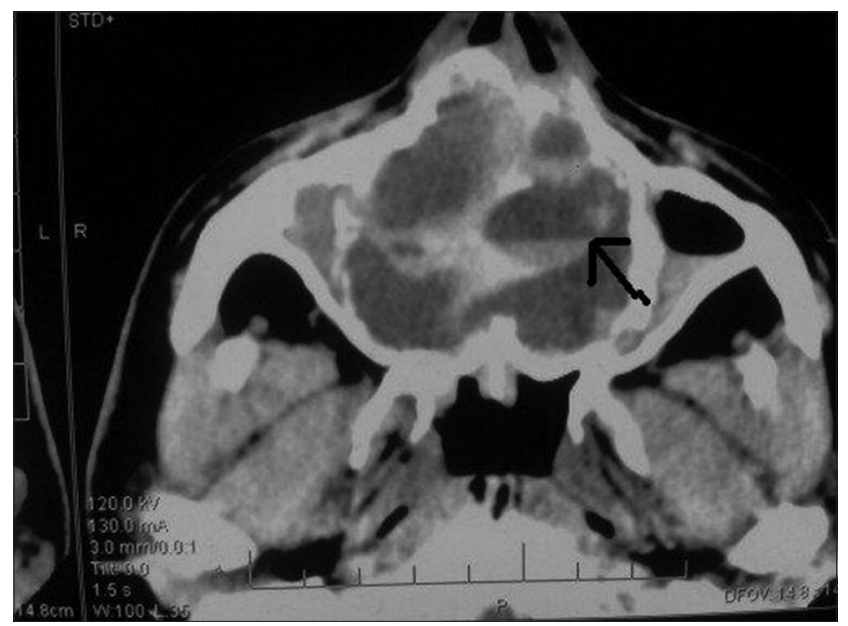

Figure 1: Axial section of noncontrast computerized tomography of nasopharyngeal region. There is an expansile lesion causing obliteration of right maxillary sinus. Multiple fluid levels are seen (black arrow)

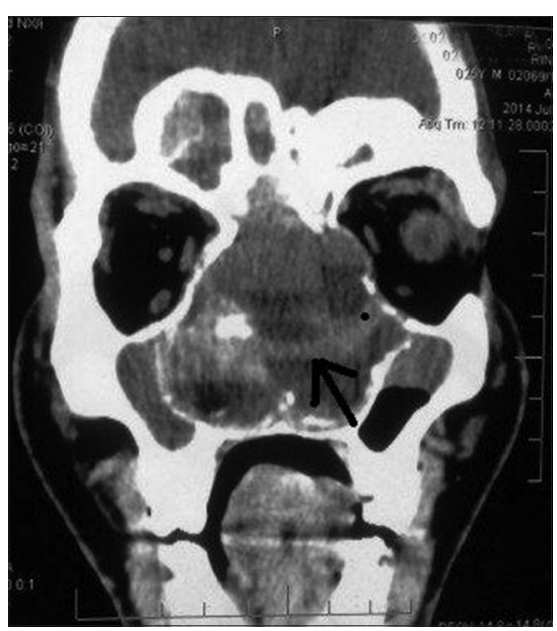

Figure 3: Noncontrast computerized tomography coronal section shows an expansile "blown out" lesion in the nasopharyngeal region with multiple fluid levels (black arrow). There is no destruction of the bone

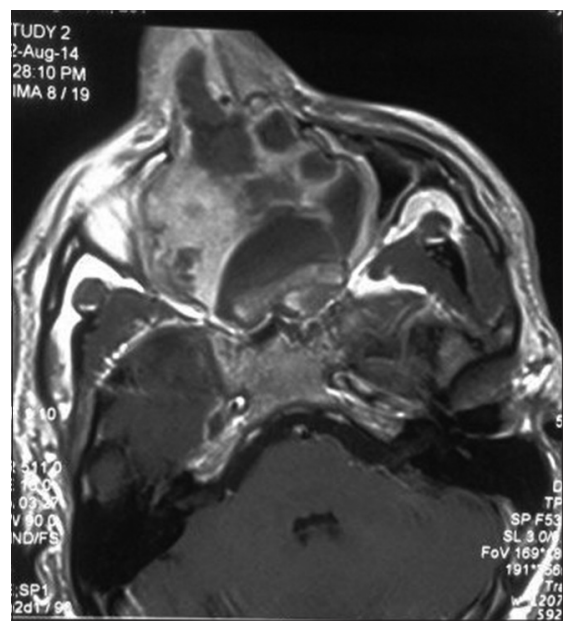

Figure 5: Contrast enhanced magnetic resonance T1-weighted axial section shows variable septal enhancement along with collapse of the right maxillary sinus

osteoclast giant cells." These are usually found within bones with high venous pressure and marrow content. Sixty-five percent are primary or simple, and 35\% are secondary in

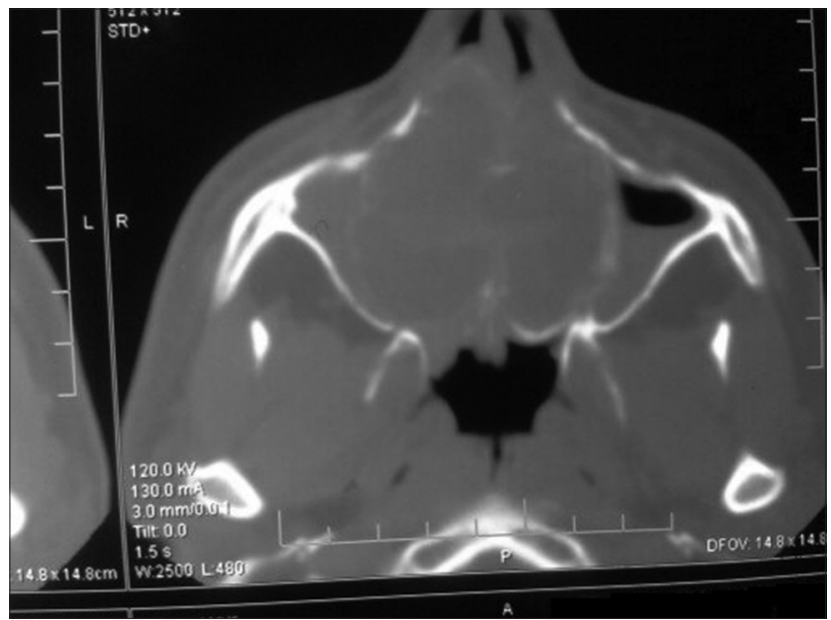

Figure 2: Axial section of noncontrast computerized tomography of nasopharyngeal region in bone window. There is expansion and thinning of the bony cortex. Both the pterygoid plates are intact

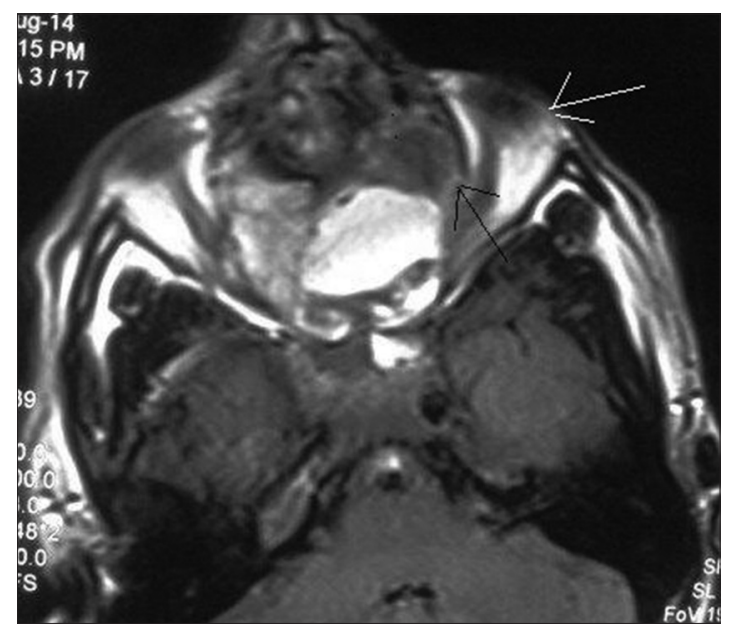

Figure 4: Magnetic resonance T1-weighted axial section of the nasopharyngeal region shows an expansile aneurysmal bone cyst with heterogenous intensities due to blood within the lesion. Multiple fluid levels are seen within the lesion. There is bulging of the left eyeball (white arrow) because of the mass effect (black arrow)

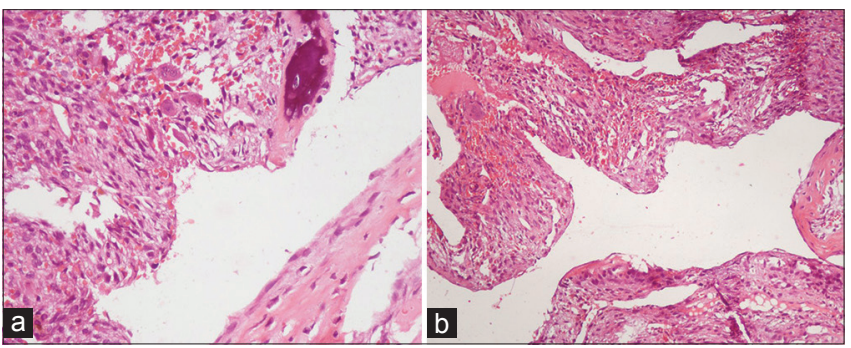

Figure 6: (a) Low power photomicrograph showing cystic spaces among fibrous tissue $(\mathrm{H}$ and $\mathrm{E}, \times 200)$; (b) high power photomicrograph showing cysts without endothelial lining and wall containing giant cells and reactive bone formation $(\mathrm{H}$ and $\mathrm{E}, \times 200)$

nature as per Bonakdarpour et al. ${ }^{[3]}$ Primary $\mathrm{ABC}$ has a high level of accuracy of diagnosis with radiological studies, diagnosis, but only $20 \%$ of secondary lesions can be diagnosed radiographically. It is usually found in individuals $<20$ years old. There is no predilection for gender, but a 2:1 ratio in female to male has been recorded. $A B C$ in the maxillary region may remain undiagnosed for a long period secondary to its gradual course as occurred in 
our case. A patient will generally report to the hospital after becoming symptomatic. The underlying pathology of giant cell tumor is well-known in secondary types of $\mathrm{ABC}$. It is always diagnosed when the patient undergoes three dimensional imaging modalities such as CT and MRI. Radiographically, ABC gives the impression of a "blown out" or "ballooned" appearance of the underlying bone. CT and MRI demonstrate classic fluid-level images, but histological examination remains the hallmark for the diagnosis. The solid component of the lesion can be visualized on CT and MRI, which further indicates a secondary nature. Multiple areas of high intensity surrounded by hypointense rings are observed on T1-weighted and T2-weighted images. These represent the different ages of blood in the lesion. ${ }^{[4]}$ There should be close collaboration between radiologist and pathologist when making the diagnosis, as a small specimen may miss the associated findings.

Surgical excision with enucleation remains the best treatment. The path to surgery depends upon the extent of involvement and its nature. Other potential options for treatment include percutaneous sclerotherapy, therapeutic embolization, curettage, block resection and reconstruction, radiotherapy and systemic calcitonin therapy. ${ }^{[5]}$ There have some cases of spontaneous healing. ${ }^{[6]}$

In conclusion, $\mathrm{ABC}$ of hidden areas such as the maxilla as in our case poses a great diagnostic and management dilemma for the clinician. CT and MRI are quite helpful modalities in guiding the management path. This further helps in ruling out intracranial extension. The radiological modalities further help the anesthesiologist in guiding intubation and determining the level of risk during surgery.

\section{REFERENCES}

I. Suzuki F, Fukuda S, Yagi K, Chida E, Inuyama Y. A rare aneurysmal bone cyst of the maxillary sinus: a case report. Auris Nasus Larynx 200I;28:SI3I-7.

2. Guzmán GP, Baeza OA, Araya OJ, Roa SJ, Brevis OL, Torres LP. Aneurysmal bone cyst of the maxilla. Report of one case. Rev Med Chil 2005; 133:1355-60.

3. Bonakdarpour A, Levy WM, Aegerter E. Primary and secondary aneurysmal bone cyst: a radiological study of 75 cases. Radiology 1978;126:75-83.

4. Pahade J, Sekhar A, Shetty SK. Imaging of malignant skeletal tumors. In: Blake MA, Kalra MK, editors. Imaging in Oncology. New York: Springer-Verlag; 2008. p. 375.

5. Rai AT, Collins JJ. Percutaneous treatment of pediatric aneurysmal bone cyst at $\mathrm{CI}$ : a minimally invasive alternative: a case report. AJNR Am J Neuroradiol 2005;26:30-3.

6. Malghem J, Maldague B, Esselinckx W, Noel H, De Nayer P, Vincent A. Spontaneous healing of aneurysmal bone cysts. A report of three cases. J Bone Joint Surg Br 1989;71:645-50.

How to cite this article: Sharma BB, Ramchandran P, Sharma S, Sharma S. Aneurysmal bone cyst of the maxilla rare presentation with radiological and pathological correlation. Plast Aesthet Res $2015 ; 2: 73-5$.

Source of Support: Nil, Conflict of Interest: None declared.

Received: 21-09-2014; Accepted: 17-10-2014 